\title{
Prevalence of Trichomoniasis Among Women in Kermanshah, Iran
}

\author{
Naser Nazari ${ }^{1}$; Maryam Zangeneh ${ }^{2,}$; Fatemeh Moradi $^{3}$; Arezoo Bozorgomid $^{3}$ \\ ${ }^{1}$ Department of Parasitology and Mycology, Medical School, Kermanshah University of Medical Sciences, Kermanshah, IR Iran \\ ${ }^{2}$ Department of Obstetrics and Gynecology, High Risk Pregnancy Research Center, Imam Reza Hospital, Kermanshah University of Medical Sciences, Kermanshah, IR Iran \\ ${ }^{3}$ Medical School, Kermanshah University of Medical Sciences, Kermanshah, IR Iran \\ ${ }^{*}$ Corresponding Author: Maryam Zangeneh, Department of Obstetrics and Gynecology, High Risk Pregnancy Research Center, Imam Reza Hospital, Kermanshah University of Medi- \\ cal Sciences, Kermanshah, IR Iran. Tel: +98-9188323419, E-mail: mzangene.59710@yahoo.com \\ Received: September 14, 2014; Revised: November 24, 2014; Accepted: December 16, 2014
}

\begin{abstract}
Background: Trichomoniasis, caused by Trichomonas vaginalis, is the most common sexually transmitted disease (STD) in the world with estimates of 7-8 million infections annually in the United States and 180 million globally.

Objectives: This study aimed to determine the prevalence and risk factors for T. vaginalis infection in women who attended the Clinic of Obstetrics and Gynecology in Kermanshah, Iran.

Patients and Methods: This cross-sectional study was performed on all women aged 15 to 65 years who attended the clinic of obstetrics and gynecology, Imam Reza Hospital, Kermanshah, west of Iran, between September 2011 and July 2012. Vaginal secretion samples were examined by the Dorset culture medium and wet-mount methods. Demographic and personal information were also collected via questionnaires and the data were analyzed using chi-square and Fisher exact tests.

Results: A total of 600 women were screened for T. vaginalis infection and this infection was diagnosed in 9 (1.5\%) and 13 cases (2.1\%) using the wet mount and culture methods, respectively. The age and husband occupation were significantly associated with an increased risk of trichomoniasis in the infected cases $(\mathrm{P}<0.05$ and $\mathrm{P}<0.001$, respectively); other variables, including the patients' occupation and education level appeared to be positively (but not significantly) correlated with infection $(\mathrm{P}>0.05)$.

Conclusions: The prevalence of T. vaginalis infection in the study population was low. Diagnosis of trichomoniasis based on only clinical symptoms is not efficient for treatment decision. Based on our results, we suggest using the culture method on all the suspected cases even if the wet smear is negative.
\end{abstract}

Keywords: Trichomoniasis; Epidemiology; Diagnosis; Iran

\section{Background}

Vaginal infection is one of the most common conditions for which women seek treatment. It is usually characterized by vaginitis, urethritis, vaginal discharge, vulvar itching and irritation (1). Trichomonas vaginalis infection is the most common nonviral sexually transmitted infections (STI) in the world with estimates of 7-8 million infections annually in the United States and 180 million globally $(2,3)$. Asymptomatic trichomoniasis has been associated with preterm delivery and delivering low-birth weight infants. It may also increase the risk of acquisition and transmission of human immunodeficiency virus (HIV) and herpes simplex virus type 2 infection (4). It is estimated that up to $50 \%$ of the infected women are asymptomatic (5), emphasizing the importance of screening and laboratory tests in detection of trichomoniasis. Wet mount and culture techniques are the most widely used methods for the diagnosis of T. vaginalis, with a sensitivity of $51 \%$ to $66 \%$ and $75 \%$ to $85 \%$, respectively $(6,7)$. The culture technique was considered the most accurate method (the traditional gold standard) for the diagnosis of trichomoniasis. Therefore, using culture in addition to wet mount is suggested specially when $T$. vaginalis is suspected but not seen on the wet mount (8). In Iran, the prevalence rates of trichomoniasis in woman have varied depending on the population studied and the diagnostic methods used or health-seeking behaviors (9-11).

\section{Objectives}

The purpose of this study was to assess the prevalence of T. vaginalis infection and its associated risk factors among women who attended the Clinic of Obstetrics and Gynecology in Kermanshah, Iran. Furthermore, in this study we evaluated the utility of direct microscopic examination for the diagnosis of trichomoniasis and compared it with culture technique.

\section{Patients and Methods}

A cross-sectional study was performed on 600 women aged 15 to 65 years attending the Obstetrics and Gynecology Clinic of Imam Reza Hospital in Kermanshah, Iran, from September 2011 to July 2012. The Educational, Research and Treatment Center of Imam Reza Hospital is the largest center among referral centers in Kermanshah Province, west of Iran. Based on the previous studies in Iran mentioning the prevalence of 6 percent for

Copyright (C) 2015, Iranian Red Crescent Medical Journal. This is an open-access article distributed under the terms of the Creative Commons Attribution-NonCommercial 4.0 International License (http://creativecommons.org/licenses/by-nc/4.0/) which permits copy and redistribute the material just in noncommercial usages, provided the original work is properly cited. 
Nazari $N$ et al.

trichomoniasis women (9-11), with confidence level of $95 \%$ and a margin of error of $2 \%$, the minimum sample size required was 542 subjects, which was increased to 600 to allow for dropouts. Women attending the clinic were invited to participate in the study and a letter of consent was requested from all study participants. The age range of women in the study was 15 to 65 years old. We excluded 12 cases from the study because of pregnancy situation and incomplete data.

Two vaginal swab specimens were collected from each participant. The sample tubes containing $2 \mathrm{~mL}$ of dextrose saline solution were immediately transported to parasitology laboratory, Kermanshah University of Medical Sciences (Kermanshah, Iran) within 3 hours after collection. The specimens were subjected to wet mount examination and culture techniques by using light microscope and Dorset culture medium, respectively. The cultures were kept in the incubator at $37^{\circ} \mathrm{C}$ for 3 days and examined for growth on days 1, 2, and 3. The clinical signs (existence of discharge, itching, dysuria, dyspareunia, inflammation of the genital tract, foul odor) were collected through interview and clinical examination was performed by one gynecologist and the $\mathrm{pH}$ of vaginal content was determined by Litmus paper; the color change was compared with a $\mathrm{pH}$ standard color chart. The vaginal $\mathrm{pH}$ above 4.5 generally is an indicative of trichomoniasis. Socio-demographic information (age, education, patient occupation, and husband's occupation, urban or rural residency) of the subjects were collected using questionnaires. The chi- square and Fisher exact tests were used to analyze the data at a significance level of 0.05 using SPSS 13 (version 13.5; SPSS Inc, Chicago, IL, USA). The present study was approved by Ethical Committee of Kermanshah University of Medical Sciences.

\section{Results}

This study was conducted on a total of 600 women attending obstetrics and gynecology clinic. The age range of the participants was 15 to 65 years. Majority (36.3\%) of them had high school level of education and $20 \%$ were illiterate (Table 1).

Among a total of 600 participants, 78\% were from urban and $22 \%$ from from rural areas. Most of the women (69.6\%) were housewife and 30.4\% had official job. Most of the husbands of the infected individuals with T. vaginalis were drivers $(\mathrm{P}=0.001)$, but no significant associations were observed between trichomoniasis and occupation of women $(\mathrm{P}=0.122)$. Twelve of those who were infected were housewives and one was clerk. Trichomonas vaginalis was detected in 13 out of the 600 participants $(2.1 \%)$ by using culture method as gold standard, whereas only 9 out of the 13 infected women were found positive using the wet mount technique (1.5\%). There was no statistical correlation between clinical and parasitological diagnosis methods $(\mathrm{P}=0.18)$. The highest infection rate was in the age group of 21-35 years (12 cases). Most of the infected individuals with $T$. vaginalis $(8.13,61 \%)$ had no education $(\mathrm{P}=0.002)$.

\begin{tabular}{|c|c|c|c|c|}
\hline Characteristic & Trichomoniasis-Positive & Trichomoniasis-Negative & Total & P Value \\
\hline Age, y & & & & 0.109 \\
\hline$\leq 20$ & $0(0)$ & $25(100)$ & 25 & \\
\hline $21-35$ & $12(3.3)$ & 348 (96.7) & 360 & \\
\hline $36-50$ & $1(1.03)$ & 100 (98.97) & 101 & \\
\hline$\geq 50$ & $0(0)$ & $114(100)$ & 114 & \\
\hline Occupation & & & & 0.122 \\
\hline Housewife & $12(2.9)$ & $406(97.1)$ & 418 & \\
\hline Official job & $1(0.5)$ & $181(99.5)$ & 182 & \\
\hline Residency & & & & 0.744 \\
\hline Urban & $11(2.3)$ & $458(97.7)$ & 469 & \\
\hline Rural & $2(1.5)$ & $129(98.5)$ & 131 & \\
\hline Education & & & & 0.002 \\
\hline Illiterate & $8(6.7)$ & $112(93.3)$ & 120 & \\
\hline Primary & $3(1.7)$ & $169(98.3)$ & 172 & \\
\hline High school & $2(0.9)$ & $216(99.1)$ & 218 & \\
\hline College & $0(0)$ & $90(100)$ & 90 & \\
\hline Total & $13(2.2)$ & $587(97.8)$ & & \\
\hline
\end{tabular}

\footnotetext{
${ }^{a}$ data are presented as No. (\%).
} 
Nazari $N$ et al.

Symptoms varied among the patients; however, the most and the least complaints were itching (75.5\%) and vaginitis (19.7\%), respectively. Fifty percent of the women with trichomoniasis declared vaginal discharge, $29.6 \%$ bad genital odor, 3.3\% itching, 4.4\% dyspareunia and $6.6 \%$ a burning sensation in the genitalia. In this study, the highest prevalence of trichomoniasis was observed in the cases with $\mathrm{pH}=5.6-6$. Our study showed a significant correlation between $T$. vaginalis and watery, frothy vaginal discharge $(P=0.001)$. There was no statistically significant difference related to contraception methods used by the participants $(\mathrm{P}=0.322)$. However, the condom use is associated with lower prevalence of trichomoniasis and other sexually transmitted diseases (STDs) (12).

\section{Discussion}

In the present study, the prevalence of T. vaginalis infection was found lower than the previous studies in Iran (2.1\%), such as in Shiraz the trichomoniasis prevalence of $11.2 \%$ in vulnerable household women who were covered by the Imam Khomeini Relief Foundation insurance scheme was reported (10), while the prevalence in nonpregnant women referred to health centers were as follows: 9.2\% in Tabriz (13), 4.8\% in Zanjan (14) and in Tehran $3.2 \%(15)$.

The prevalence of trichomoniasis in rural area of Southern Mozambique (16), Palestine (17), Argentina (18) and USA (19) were reported $31 \%, 28.1 \%, 4 \%$, and $13 \%$, respectively. Therefore, it can be concluded that the Trichomonas prevalence varies in different societies, which might be due to different factors, such as hygiene behaviors and sociodemographic characteristics and diagnostic tests used. In addition, one possible explanation of the lower prevalence of trichomoniasis among Iranian population is due to Islamic quotes that prohibit nonmarital sex and homosexuality. Moreover, Chalechale et al. stated that the lower incidence of infection may have been related to universal male circumcision in Iran (9). In this study, the women aged 21 to 35 years had the highest infection rate. This finding is consistent with the results from several previous studies $(20,21)$. This may be due to biologic changes in older women as one of the predisposing factors for the parasite growth or longer duration of infectiousness and resistance to treatment. Although the prevalence of trichomoniasis in the urban dwellers was higher than the rural dwellers (11/13), the percentage of urban and rural participation was $78 \%$ and $22 \%$, respectively. This difference is difficult to interpret indeed whether the prevalence of disease is more in urban or rural areas.

Our study showed a significant correlation between $T$. vaginalis and women whose husbands were unemployed or drivers as compared to the others $(6 / 13, \mathrm{P}<0.001$, df $=1$ ). Other variables that appeared to be positively correlated with infection, although not statistically significant, included patients' occupation and education level. The prevalence rate of $T$. vaginalis among intra uterine device (IUD) users (8.3\%) was higher than nonusers (4.1\%). In some studies, the higher prevalence of trichomonas infection in IUD users was associated with T. vaginalis infection, but this is not consistent with our results as we found no significant correlation $(22,23)$. According to our result, no significant association was seen between condom use and infection; however, several studies have shown that condom users and/or their partners have a lower frequency of STIs than persons who do not use condoms $(12,24)$.

Trichomonas vaginalis was detected in 13 out of the 600 participants (2.1\%) using the culture methods, whereas only 9 out of the 13 infected women were found to be positive using the wet mount technique (1.5\%). These results were similar to other studies that lower prevalence rates were shown using microscopic examinations. Rezaeian et al. reported prevalence rates of $3.2 \%$ and $2.6 \%$ in women attended to the sexually transmitted disease clinic using the culture method and the wet mount, respectively (15). Radonjic et al. in Belgrade reported similar results to our data, $10.5 \%$ and $7 \%$ for culture and microscopic examinations, respectively (25). Some authors report that the culture method has a higher sensitivity (75\% to $85 \%$ ) than the wet mount and a specificity of $100 \%$ (7), as very few organisms are required for a positive result, but this method is expensive and needs a prolonged time for diagnosis. Therefore, we suggest culturing method when T. vaginalis is suspected, but not seen on wet mount or whenever the prevalence of infection is high in the population. Even though itching and vaginitis were the frequent complaints among the investigated women, they were not associated with trichomoniasis in this study. Nevertheless, other genital complaints, such as watery, frothy vaginal discharge were associated with T. vaginalis $(\mathrm{P}<0.001)$.

To the best of our knowledge, no study has been done on the use of wet mount and culture methods for the diagnosis of trichomoniasis among women in Kermanshah City, Iran. Nevertheless, our study has certain limitations and thus more studies need to be done with larger sample size and with considering more criteria in future. First, we studied women who attended STD clinics that it may not be generalized to general population and therefore might not reflect the real prevalence of this infection and associations between the risk factors that needs to include more cases and even including apparent healthy women in future. Second, we did not assess participants for antibiotic-resistant $T$. vaginalis infection, although it was asked in the questionnaire, a more accurate method should be used to assess this issue in future. In addition, trichomoniasis infection is usually asymptomatic in men and they can transmit the parasite to their partner during sexual intercourse. Therefore, the question remains about the prevalence rate of infection in men in Kermanshah City that is needed to be studied in future.

Based on the above findings, it can be concluded that the diagnosis of T. vaginalis should not be based on symptoms alone and at least the use of wet mount prepara- 
tion should be recommended for diagnosis of $T$. vaginalis infection. Moreover, our study indicated that trichomoniasis infection is not noticeable, as much as other pathogens, such as nongonococcal urethritis, candidiasis, gonococcal urethritis, syphilis, etc and thus special attention should be paid to this matter.

\section{Acknowledgements}

We would like to thank the Obstetrics and Gynecology Clinic staff of Imam Reza Hospital (Kermanshah, Iran) for their kind cooperation.

\section{Authors' Contributions}

Naser Nazari, Maryam Zangeneh: Study concept and design; Fatemeh moradi: Acquisition of data; Arezoo Bozorgomid: Analysis and interpretation of data; Naser Nazari, Maryam Zangeneh: Drafting of the manuscript, critical revision of the manuscript for important intellectual content; Naser Nazari, Maryam Zangeneh, Arezoo Bozorgomid: Statistical analysis; Naser Nazari, Maryam Zangeneh: Administrative, technical, and material support; Naser Nazari, Maryam Zangeneh:Study supervision.

\section{Funding/Support}

This study was funded by Kermanshah University of Medical Sciences, Kermanshah, Iran.

\section{References}

1. Schwebke JR, Burgess D. Trichomoniasis. Clin Microbiol Rev. 2004;17(4):794-803.

2. World Health Organization. Trichomonas.; 2010. http://search. who.int/search

3. Cates WJ. Estimates of the incidence and prevalence of sexually transmitted diseases in the United States. American Social Health Association Panel. Sex Transm Dis. 1999;26(4 Suppl):S2-7.

4. McClelland RS, Sangare L, Hassan WM, Lavreys L, Mandaliya K, Kiarie J, et al. Infection with Trichomonas vaginalis increases the risk of HIV-1 acquisition. J Infect Dis. 2007;195(5):698-702.

5. Heine P, McGregor JA. Trichomonas vaginalis: a reemerging pathogen. Clin Obstet Gynecol. 1993;36(1):137-44.

6. Wiese W, Patel SR, Patel SC, Ohl CA, Estrada CA. A meta-analysis of the Papanicolaou smear and wet mount for the diagnosis of vaginal trichomoniasis. Am J Med. 2000;108(4):301-8.

7. Borchardt KA, Zhang MZ, Shing H, Flink K. A comparison of the sensitivity of the InPouch TV, Diamond's and Trichosel media for detection of Trichomonas vaginalis. Genitourin Med. 1997;73(4):297-8.

8. Swygard H, Miller WC, Kaydos-Daniels SC, Cohen MS, Leone PA, Hobbs MM, et al. Targeted screening for Trichomonas vaginalis with culture using a two-step method in women presenting for STD evaluation. Sex Transm Dis. 2004;31(11):659-64.
9. Chalechale A, Karimi I. The prevalence of Trichomonas vaginalis infection among patients that presented to hospitals in the Kermanshah district of Iran in 2006 and 2007. Turk J Med Sci. 2010;40(6):971-5.

10. Maharlouei N, Barooti E, Sharif F, Hosseini H, Lankarani KB. Prevalence and risk factors of reproductive tract infections among a defined population of Iranian women. Sex Health. 2013;10(4):311-

11. Rabiee S, Fallah M, Zahabi F. Frequency of Trichomoniasis in Patients Admitted To Outpatient Clinics in Hamadan (2007) and Relationship between Clinical Diag-nosis and Laboratory Findings. J Res Health Sci. 2010;10(1):31-5.

12. Holmes KK, Levine R, Weaver M. Effectiveness of condoms in preventing sexually transmitted infections. Bulletin World Health Organiz. 2004;82(6):454-61.

13. Gavgani AM, Namazi A, Ghazanchaei A, Alizadeh S, Sehhati F, Rostamzadeh S, et al. Prevalence and risk factors of trichomoniasis among women in Tabriz. Arch Clin Infect Dis. 2008;3(2)

14. Bahram A, Hamid B, Zohre T. Prevalence of bacterial vaginosis and impact of genital hygiene practices in non-pregnant women in zanjan, iran. Oman Med J. 2009;24(4):288-93.

15. Rezaeian M, Vatanshenassan M, Rezaie S, Mohebali M, Niromand $\mathrm{N}$, Niyyati M, et al. Prevalence of Trichomonas vaginalis using parasitological methods in Tehran. Iran J Parasitol. 2009;4(4):43-7.

16. Menendez C, Castellsague X, Renom M, Sacarlal J, Quinto L, Lloveras B, et al. Prevalence and risk factors of sexually transmitted infections and cervical neoplasia in women from a rural area of southern Mozambique. Infect Dis Obstet Gynecol. 2010;2010.

17. Houso Y, Farraj MA, Ramlawi A, Essawi T. Detection of Trichomonas vaginalis in Vaginal Swab Clinical Samples from Palestinian Women by Culture. ISRN Microbiol. 2011;2011:872358.

18. Perazzi BE, Menghi CI, Coppolillo EF, Gatta C, Eliseth MC, de Torres RA, et al. Prevalence and comparison of diagnostic methods for Trichomonas vaginalis infection in pregnant women in $\mathrm{Ar}$ gentina. Korean J Parasitol. 2010;48(1):61-5.

19. Helms DJ, Mosure DJ, Metcalf CA, Douglas JJ, Malotte CK, Paul SM et al. Risk factors for prevalent and incident Trichomonas vaginalis among women attending three sexually transmitted disease clinics. Sex Transm Dis. 2008;35(5):484-8.

20. Matini M, Rezaie S, Mohebali M, Maghsood A, Rabiee S, Fallah M, et al. Prevalence of Trichomonas vaginalis Infection in Hamadan City, Western Iran. Iran J Parasitol. 2012;7(2):67-72.

21. Salmani R, Baghchesaraie H, Amini B. Prevalence of Trichomonas Vaginalis Infection Among Women Refered to Laboratories in Zanjan, 2010. J Res Develop Nurs Midwife. 2012;9(1):69-75.

22. Demirezen S. Trichomonas vaginalis in vaginal smears of women using intrauterine contraceptive device. Cent Eur J Public Health. 2001;9(4):176-8.

23. Joesoef MR, Karundeng A, Runtupalit C, Moran IS, Lewis JS Ryan CA. High rate of bacterial vaginosis among women with intrauterine devices in Manado, Indonesia. Contraception. 2001;64(3):169-72.

24. Crosby RA, Charnigo RA, Weathers C, Caliendo AM, Shrier LA Condom effectiveness against non-viral sexually transmitted infections: a prospective study using electronic daily diaries. Sex Transm Infect. 2012;88(7):484-9.

25. Radonjic IV, Dzamic AM, Mitrovic SM, Arsic Arsenijevic VS, Popadic DM, Kranjcic Zec IF. Diagnosis of Trichomonas vaginalis infection: The sensitivities and specificities of microscopy, culture and PCR assay. Eur J Obstet Gynecol Reprod Biol.2006;126(1):116-20. 